
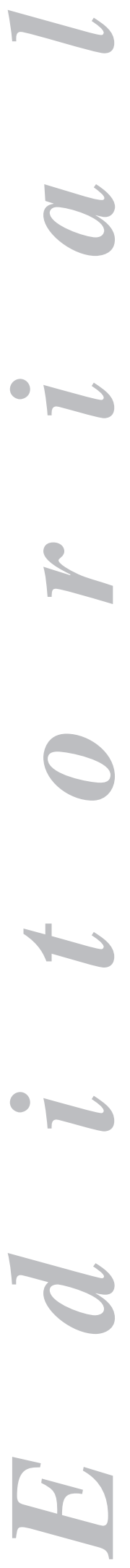

\section{Interleukins and colon cancer}

Tumor development is interpreted as a phenomenon escaping immunosurveillance. Furthermore, tumor tissue seems to have little immunogenic power. However, a variable proportion of lymphocytic infiltration may be identified in tumors, and colorectal cancer is an example of a tumor with considerable T-cell infiltration overall, which seems to be associated with a better prognosis $(1,2)$. In fact, in both human gastric and human rectal cancers, infiltrating lymphocytes are predominantly of the Th1 and cytotoxic-1 T-cell types, with gamma-interferon predominating over other Th2-dependent cytokines including IL-4, which may be important for anti-tumoral immune response (3). For example: while studying these immunologic issues in human colorectal cancer, tumors expressed CCR5 -in association with its ligand: RANTES- and CXCR3 (Th1 markers) in marginal tumor infiltrates; CCR4 (Th2 marker) expression was rarer, which would indicate recruitment of Th1 cells and both CD8 and CD4+ cytotoxic T cells towards tumoral tissue (4).

The role of specific cytokines -including chemokines- in immune defense against tumor development and extension is being currently assessed by multiple studies oriented towards the design of effective immunobiologic anti-tumoral therapies. Most of them are experimental, albeit a number of therapeutic attempts in humans already exist in view of the not-so-good prospects of surgery, radiotherapy, and chemotherapy for advanced-stage tumors.

However, specific cytokines may on occasion encourage tumor growth and particularly tumor spread, as is the case with IL-1 $\beta$ and TNF $\alpha$, which are released for instance during surgery and may activate adhesion molecules (ICAM-1 and VCAM-1, and their ligands LFA-1 and VLA-4) at the lung's vascular endothelium, thus promoting pulmonary metastasis (5). In a study carried out in patients with colon carcinoma serum IL-6, TNF $\alpha$ and PCR levels were seen to correlate with prognosis in terms of tumor mass and survival (6), while IL-6 and TGF $\alpha$ levels, and to a lesser extent TNF $\alpha$ levels, were seen to correlate with quality of life (7). Regarding the liver, experimental studies have suggested that Kupffer cells may well release IL-6, IL-10 and TNF $\alpha$ when stimulated by carcinoembrionary antigen (CEA). This may induce ICAM-1 expression and a greater potential for neoplastic cell retention within sinusoids, thus resulting in colon carcinoma metastases to the liver (8).

Regarding cytokines, IL-2 and IL-12 are most involved in immunobiological anti-tumoral response (9). As for IL-2, its local administration in transplanted colon carcinoma induced a macrophage response with tumor necrosis, while systemic administration had no effect other than notable adverse events (10). Other authors identified a rejection of specific colon tumors in mice following vaccination using dendritic cells loaded with anti-idiotype $3 \mathrm{H} 1$ antibodies (which mimics carcinoem- 
brionary antigen, CEA). This is consistent with the induction of antigen-specific CD8+ T cells that may express IL-2, gamma-interferon, and TNF $\alpha$ (11). One study attempted immune therapy using IL-2-activated lymphocytes from the excised tumor in patients undergoing hepatectomy for colon carcinoma metastases, together with a systemic infusion of IL-2. Besides side effects, only a small percentage of patients responded during follow-up for 5 years (12).

In contrast, many positive results have been obtained with IL-12, as well as with IL-23 and IL-27, both of them belonging in the family of the former cytokine $(13,14)$. Indeed, IL-12 is a heterodimeric cytokine primarily released by antigenpresenting cells (macrophages, dendritic cells, and B lymphocytes), which promote proliferation and activation of NK and cytotoxic T cells. The anti-tumoral mechanism of action of IL-12 is not clearly understood, but seems to depend on the activation of these cells in addition to their anti-angiogenic and angiostatic effects. Considering also its toxic effects when systemically administered, vectors -particularly adenoviral- are being used to deliver this cytokine within the tumor or tumor-invaded tissue, thus avoiding systemic effects. For example, IL-12 gene transfection to an adenoviral vector in the mouse liver provided protection against primary and metastatic tumors. Furthermore, the effect was seen to be mediated by NK cells, which were severely activated regarding cytolitic activity and gamma-interferon production, with the latter being seemingly a major mediator of anti-tumoral protection. Adoptive transference of NK cells from previously transfected mice similarly provided protection against colorectal cancer metastasis to the liver (15). Another experiment in rats with multiple experimental colon cancer metastases to the liver included the intraportal injection of IL-12 in association with a retroviral vector, which resulted in significant reduction of tumor bulk, together with neoplastic tissue infiltration by CD8+ and NKT T cells; the latter's anti-angiogenic effects were highlighted (16). Intralesional inoculation of an HSV mutant for immunogenesis in an experimental colon tumor resulted in a local immune response that was increased by the local or systemic administration of recombinant IL-12, with a complete regression of inoculated tumors and metastases in 67 and 79\%, respectively, of animals, which opens up new research perspectives into the treatment of metastatic disease (17). An oligodeoxynucleotide with immunogenic effects (CpG ODN) induced necrosis and lymphocytic infiltration in experimental colon tumors; mouse survival increased, and the expression of specific cytokines such as IL-6 and GMC-SF, as well as the expression of a number of chemokines (CXCL1, CCL2, and CCL3), which may modulate tumor growth by affecting angiogenesis, immune response activation and/or cell proliferation, was demonstrated (18). NK-cell activation has also been studied with the administration of autologous HSPs (heat shock proteins) from patients with colorectal cancer, which increase IL-12 production and exert cytotoxic effects (19).

Other studies (20) show how intradermically inoculated murine colon tumors are eradicated by the intralesional administration of IL-12-producing dendritic cells (using "in vitro" transfection with adenoviral vectors); this seemed to be mediated by CD8+ T cells, but CD4+ and NK cells, which are stimulated to release gamma-interferon as an anti-tumoral mediator, were also seen to play a role. This effect increased upon the systemic administration of anti-CD137 (4-1BB) monoclonal agonist antibodies, as CD137 is an antigen of both activated T cells and NK cells. Regarding this synergic effect of IL-12 and anti-CD137 agonist antibodies, other experimental studies (21) demonstrate that NK cells play a vital role, as through gamma-interferon production they induce the activation and ex- 
pansion of dendritic cells, which deserves investigation in relation to anti-tumoral immune therapy.

In a human Phase I trial, intratumoral administration (for primary or metastatic liver tumors) of an adenoviral vector coding for human IL-12 genes increased tumor infiltration with $\mathrm{T}$ lymphocytes (CD4 and CD8+), albeit only a moderate antitumoral effect was achieved, which was somewhat larger for primary tumors (22).

In this issue of our Journal, Coca et al. (23) present a study in rats with experimental colon cancer where tumor frequency and bulk were smaller in rats treated before tumor inductor administration by intraperitoneal IL-12 injection, which they associate with a higher density of NK cells within infiltrates. Concurrent IL-2 administration was not protective, possibly -as the authors themselves state- because of the smaller IL-12 dose that was administered to the combination therapy group as a result of side effects. In this respect, the option of directed immune therapy using vectors to facilitate higher drug concentrations locally, with the result of fewer systemic adverse events, should again be offered.

\section{A. Caro-Patón Gómez}

School of Medicine. Service of Digestive Diseases. Hospital Universitario Río Hortega. Valladolid, Spain

\section{REFERENCES}

1. Ropponen KM, Eskelinen MJ, Lipponen PK, Alhava E, Kosma VM, Prognostic value of tumour-infiltrating lymphocytes (TILs) in colorectal cancer. J Pathol 1997; 182: 318-24.

2. Menon AG, van Rhijn CMJ, Morreau H, Putter H, Tollenaar RAEM, van de Velde CJH, et al. Immune system and prognosis in colorectal cancer: a detailed immunohistochemical analysis. Lab Invest 2004; 84: 493-501.

3. Matsutani T, Shiiba K, Yoshioka T, Tsuruta Y, Suzuki R, Ochi T, et al. Evidence for existence of oligoclonal tumor-infiltrating lymphocytes and predominant production of T helper 1/T cytotoxic 1 type cytokines in gastric and colorectal tumors. Int J Oncol 2004; 25: 133-41.

4. Musha H, Ohtani H, Mizoi T, Kinouchi M, Nakayama T, Shiiba K, et al. Selective infiltration of CCR5 (+)CXCR3 (+) T lymphocytes in human colorectal carcinoma. Int J Cancer 2005 (in press).

5. ten Kate M, Hofland LJ, van Grevenstein WM, van Koetsveld PV, Jeekel J, van Eijck CH. Influence of proinflammatory cytokines on the adhesion of human colon carcinoma cells to lung microvascular endothelium. Int J Cancer 2004; 112: 943-50.

6. Nikiteas NI, Tzanakis N, Gazouli M, Rallis G, Daniilidis K, Theodoropoulos G, et al. Serum IL-6, TNFalpha and CRP levels in greek colorectal cancer patients: prognostic implications. World J Gastroenterol 2005; 11: 1639-43.

7. Rich T, Innominato PF, Boerner J, Mormont MC, Iacobelli S, Baron B, et al. Elevated serum cytokines correlated with altered behaviour, serum cortisol rythm, and dampened 24-hour rest-activity patterns in patients with metastatic colorectal cancer. Clin Cancer Res 2005; 11: 1757-64.

8. Thomas P, Hayashi H, Zimmer R, Forse RA. Regulation of cytokine production in carcinoembryonic antigen stimulated Kupffer cells by beta- 2 adrenergic receptors: implications for hepatic metastasis. Cancer Lett 2004; 209: 251-7.

9. Kountouras J, Zavos C, Chatzopoulos D. Therapy for colorectal cancer. NEJM 2005; 352: 1820-1.

10. Kusnierczyk H, Pajtasz-Piasecka E, Koten JW, Bijleveld C, Krawczyk K, Den Otter W. Further development of local IL-2 therapy of cancer: multiple versus single IL-2 treatment of transplanted murine colon carcinoma. Cancer Immunol Immunother 2004; 53: 445-52.

11. Saha A, Chatterjee SK, Foon KA, Primus FJ, Sreedharan S, Mohanty K, et al. Dendritic cells pulsed with an anti-idiotype antibody mimicking carcinoembryonic antigen (CEA) can reverse immunological tolerance to CEA and induce antitumor immunity in CEA transgenic mice. Cancer Res 2004; 64: 4995-5003.

12. Gardini A, Ercolani G, Riccobon A, Ravaioli M, Ridolfi L, Flamini E, et al. Adjuvant, adoptive immunotherapy with tumor infiltrating lymphocyes plus interleukin-2 after radical hepatic resection for colorectal liver metastases: 5-year analysis. J Surg Oncol 2004; 87: 46-52.

13. Chiyo M, Shimozato O, Iizasa T, Fujisawa T, Tagawa M. Antitumor effects produced by transduction of dendritic cells-derived heterodimeric cytokine genes in murine colon carcinoma cells. Anticancer Res 2004; 24: 3763-7. 
14. Hisada M, Kamiya S, Fujita K, Belladonna ML, Aoki T, et al. Potent antitumor activity of interleukin-27.CancerRes 2004; 64: 1152-6.

15. Miller G, Bleier JI, Antonescu C, Pillarisetty VG, Shah AB, Lahrs S, et al. Natural killer cell depletion confounds the antitumor mechanism of endogenous IL-12 overexpression. Int J Cancer 2004; 110: 395-402.

16. Alves A, Vibert E, Trajcevski S, Solly S, Fabre M, Soubrane O, et al. Adjuvant interleukin-12 gene therapy for the management of colorectal liver metastases. Cancer Gene Ther 2004; 11: 782-9.

17. Iizuka Y, Suzuki A, Kawakami Y, Toda M. Augmentation of antitumor immune responses by multiple intratumoral inoculations of replication-conditional HSV and interleukin-12. J Immonother 2004; 27: 92-8.

18. Sharma S, Karakousin CP, Takita H, Shin K, Brooks SP. Cytokines and chemokines are expressed at different levels in small and large murine colon-26 tumours following intratumoral injections of CpG ODN. Neoplasia 2004; 6: 523-8.

19. Pilla L, Squarcina P, Coppa J, Mazzaferro V, Huber V, Pende D, et al. Natural killer and NK-like T-cell activation in colorectal carcinoma patients treated with autologous tumor-derived heat shock protein 96. Cancer Res 2005; 65: 3942-9.

20. Tirapu I, Arina A, Mazzolini G, Duarte M, Alfaro C, Feijoo E, et al. Improving efficacy of interleukin-12-transfected dendritic cells injected into murine colon cancer with anti CD137 monoclonal antibodies and al-loantigens. Int J Cancer 2004; 110: 51-60.

21. Pan PY, Gu P, Li Q, Xu D, Weber K, Chen SH. Regulation of dendritic cell function by NK cells: mechanisms underlying the synergism in the combination therapy of IL-12 and 4-1BB activation. J Immunol 2004; 172: 4779-89.

22. Sangro B, Mazzolini G, Ruiz J, Herraiz M, Quiroga J, Herrero I, et al. Phase I trial of intratumoral injection of an adenovirus encoding interleukin-12 for advanced digestive tumors. J Clin Oncol 2004; 22: 1389-97.

23. Coca S, Enrech S, Moreno García V, Sáez MA, Gutiérrez C, Hernández JM, et al. Estudio de la respuesta antitumoral de la interleucina-12 en cáncer de colon inducido mediante 1,2-dimetilhidracina (DMH). Rev Esp Enferm Dig 2005; 97 (9): 619-28. 